\section{Gouty Tophi Presenting as Medial Patellar Plica Syndrome and Patellofemoral Disorder to Limit Knee Range of Motion}

\section{To the Editor:}

Patellofemoral disorders are the most important causes of anterior knee pain and can be treated by conservative methods, arthroscopic lateral retinacular release, and proximal and distal realignment procedures. Even medial patellar pain is more likely to be associated with patellofemoral maltracking than medial patellar plica syndrome. For anterior knee pain, an accurate history and examination confirming the diagnosis is most important to avoid unsuitable treatment.

Mechanically limiting the range of motion (ROM) of the knee joint is a rare occurrence in patients with chronic gout, which is associated with the appearance of multiple periarticular gouty tophi ${ }^{1}$ and a symptomatic tophus in the knee joint ${ }^{2-5}$. With improved medical management over the past decade, tophi deposition usually lacks obvious symptoms and seldom needs surgical intervention in the knee joint ${ }^{2-5}$. We describe a rare case of arthroscopically successful treatment for gouty tophi presenting as medial patellar plica syndrome and patellofemoral disorder causing longterm mechanical problems.

A 45-year-old male laborer complained of left anterior knee pain for 3 months with gradually limited ROM. He had had gouty arthritis without regular treatment for 13 years. After 1-month medication for acute gout attack, serum urate level had returned within normal limits $(5.9 \mathrm{mg} / \mathrm{dl})$, but the result had not been satisfactory due to persistent pain with limitation of knee ROM. Thereafter, he ignored his treatment for 1 more month. He did not recall any knee trauma that predated the initial knee symptoms. At presentation the significant physical findings of the left knee included limited ROM between $10^{\circ}$ and $100^{\circ}$ due to severe pain as well as positive WatsonJones test and patellofemoral compression grinding and gliding tests. Tenderness over the medial and lateral retinacula and positive medial patellar plica stutter test were also noted. No effusion or subcutaneous tophi could be seen.
Roentgenography showed no abnormalities or calcifications to provide diagnostic assistance beyond revealing a doubtful soft-tissue impingement in the medial patellofemoral compartment to cause tilting of the patella and equivocal osteoarthritic changes of the lateral patellofemoral compartment. Although the concentration of uric acid in the serum was $9.7 \mathrm{mg} / \mathrm{dl}$, diagnostic/therapeutic arthroscopy was performed under strong suspicion of medial patellar plica syndrome and patellofemoral disorder.

In addition to a few small tophi involving cruciate ligaments and menisci, a large tophus arising from the medial patellar plica and a proliferative synovial nodule infiltrated with tophi located in the superolateral patellofemoral compartment causing impingement were unexpectedly found at arthroscopy (Figure 1). The proliferative synovial nodule with tophi was excised and medial patellar plica excison was also performed arthroscopically. Histologic examination showed amorphous uric acid deposits surrounded by fibrous tissue and rimmed by both mononuclear histiocytes and giant cells, consistent with gouty tophi. Postoperatively, early full weight-bearing was allowed and intensive isometric quadriceps exercises were encouraged immediately. The patient had full active and passive ROM, without pain, in weeks.

Intraarticular tophi may develop early since crystal spreading is pathogenetically assumed to precipitate acute gouty attacks, but this circumstance is usually not identified by plain radiographs unless the patient has had gout for 10 to 12 years $^{6}$. Moreover, radiographs and physical examination are frequently overlooked, because intraarticular tophi and bony erosions may occur in patients without visible subcutaneous tophi, and the radiographic features are generally normal in early disease and even in this patient with chronic gout.

Under normal circumstances, the medial patellar plica is found in many knees and is not associated with any painful conditions. Once an inflammatory process is established, the normal plical tissue may hypertrophy into a truly pathological structure to cause symptoms of pain, crepitation, snapping, and popping ${ }^{7}$. Thus, there is little published information regarding gouty tophi involving the medial patellar plica and the superolateral

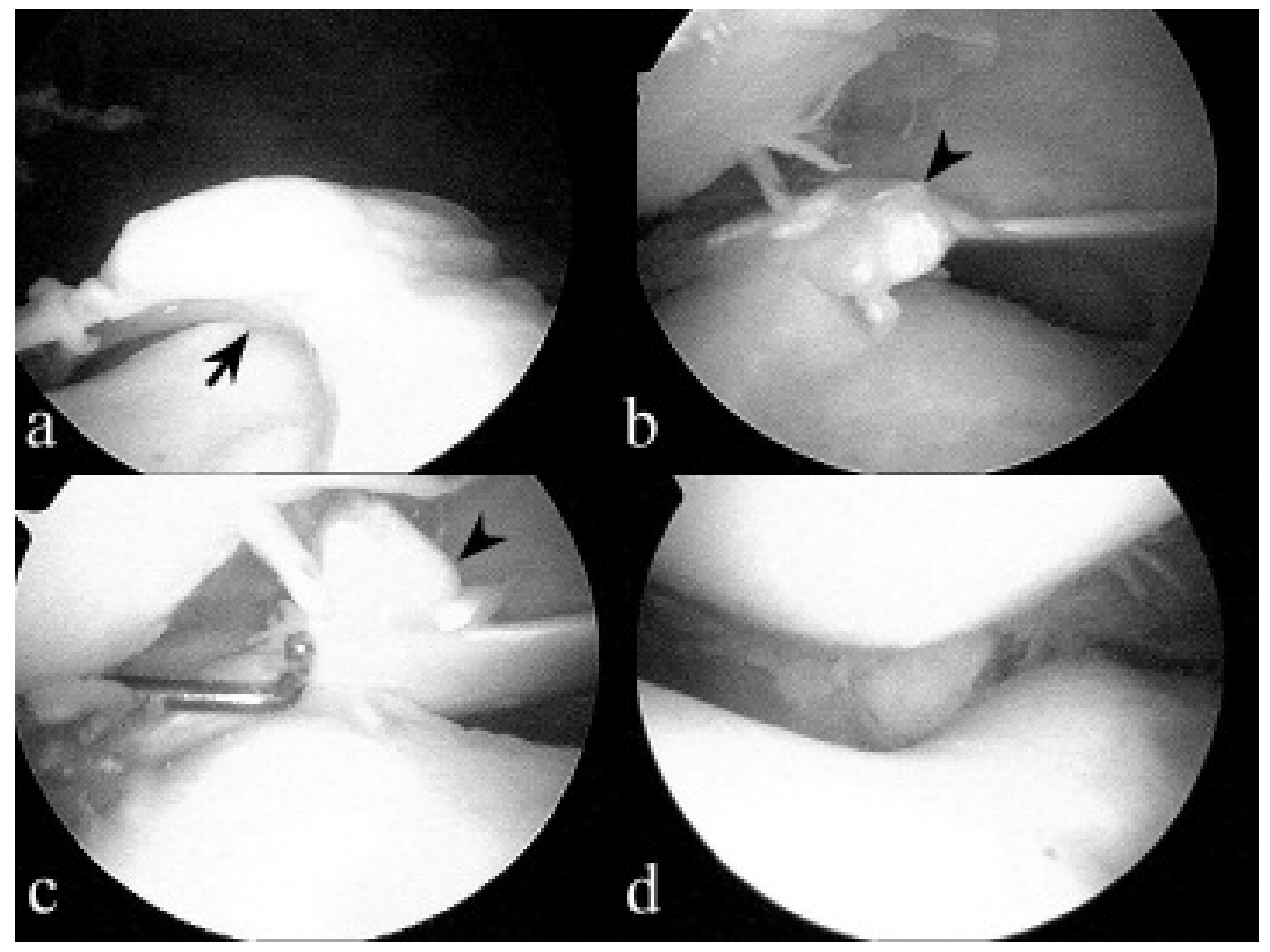

Figure 1. Intraoperative views of the 2 large tophi; (a) one in the superolateral patellofemoral compartment just over the lateral femoral condyle (arrow); (b, c) the other arising from the medial patellar plica (arrowhead). (d) The tophi and the medial patellar plica were excised and the symmetric patellofemoral congruence was also noted. 
patellofemoral compartment ${ }^{5}$. To our knowledge, this is the first report of the uncommon pathology caused by a gouty tophus hypertrophying the medial patellar plica from type $\mathrm{B}$ to type $\mathrm{C}$ and requiring excision ${ }^{8}$.

YAT-YIN LAW, MD, Department of Orthopedics, Chung Shan Medical University Hospital; KO-HUANG LUE, MD, PhD, School of Medicine, Chung Shan Medical University; KO-HSIU LU, MD, PhD, Department of Orthopedics, Chung Shan Medical University Hospital, School of Medicine, Chung Shan Medical University, 110, Section 1, Chien-Kuo N. Road, Taichung 402, Taichung, Taiwan. Address reprint requests to Dr. K-H. Lu; E-mail: cshy307@csh.org.tw

We thank Chung-Hung Tsai, MD, and Jeng-Dong Hsu, MD, Department of Pathology, Chung Shan Medical University, for their advice regarding histology, and Nieu-Tsu Han, MD, Department of Radiology, Chung Shan Medical University Hospital, for his advice regarding MRI.

\section{REFERENCES}

1. Yu KH, Lien LC, Ho HH. Limited knee joint range of motion due to invisible gouty tophi. Rheumatology 2004;43:191-4.
2. Chatterjee S, Ilaslan H. Painful knee locking caused by gouty tophi successfully treated with allopurinol. Nat Clin Pract Rheumatol 2008;4:675-9.

3. Espejo-Baena A, Coretti SM, Fernandez JM, Garcia-Herrera JM, Del Pino JR. Knee locking due to a single gouty tophus. J Rheumatol 2006;33:193-5.

4. Hammoudeh M, Siam AR, Shah S. Intra-articular tophus presenting as a loose body. Clin Rheumatol 1998;17:400-2.

5. Li TJ, Lue KH, Lin ZI, Lu KH. Arthroscopic treatment for gouty tophi mimicking an intra-articular synovial tumor of the knee. Arthroscopy 2006;22:910.

6. Yu KH. Intraarticular tophi in a joint without a previous gouty attack. J Rheumatol 2003;30:1868-70.

7. Johnson DP, Eastwood DM, Witherow PJ. Symptomatic synovial plicae of the knee. J Bone Joint Surg Am 1993;75:1485-96.

8. Sakakibara J. Arthroscopic study on iino' band (plica synovialis mediopatellaris). J Jpn Orthop Assoc 1976;50:513-22.

J Rheumatol 2010;37:3; doi:10.3899/jrheum.090889 